\title{
Dynamics of viral load and anti-SARS-CoV-2 antibodies in patients with positive RT-PCR results after recovery from COVID-19
}

\author{
Kyoung-Ho Song ${ }^{1,}{ }^{*}$, Dong-Min Kim ${ }^{2,}{ }^{*}$, Hyunju Lee ${ }^{3}$, Sin Young Ham ${ }^{1}$, Sang-Min Oh ${ }^{1}$, Hyeonju Jeong ${ }^{1}$, \\ Jongtak Jung ${ }^{1}$, Chang Kyung Kang ${ }^{4}$, Ji Young Park³, Yu Min Kang ${ }^{5}$, Ji-Yeon Kim ${ }^{6}$, Jeong Su Park ${ }^{7}$, \\ Kyoung Un Park ${ }^{7}$ Eu Suk Kim ${ }^{1}$, and Hong Bin Kim ${ }^{1}$
}

\begin{abstract}
${ }^{1}$ Department of Internal Medicine, Seoul National University Bundang Hospital, Seongnam; ${ }^{2}$ Department of Internal Medicine, Chosun University Hospital, Gwangju; ${ }^{3}$ Department of Pediatrics, Seoul National University Bundang Hospital, Seongnam; ${ }^{4}$ Department of Internal Medicine, Seoul National University Hospital, Seoul; ${ }^{5}$ Division of Infectious Diseases, Myongji Hospital, Hanyang University College of Medicine, Goyang; ${ }^{6}$ Department of Infectious Diseases, Seongnam Citizens Medical Center, Seongnam; ${ }^{7}$ Department of Laboratory Medicine, Seoul National University Bundang Hospital, Seongnam, Korea
\end{abstract}

Recently, the number of patients with coronavirus disease 2019 (COVID-19) who have tested positive for severe acute respiratory syndrome coronavirus 2 (SARSCoV-2), via the reverse transcription polymerase chain reaction (RT-PCR) test, after recovery has increased; this has caused a dilemma regarding the medical measures and policies. We evaluated the dynamics of viral load and anti-SARSCoV-2 antibodies in four patients with positive RT-PCR results after recovery. In all patients, the highest levels of immunoglobulin G (IgG) and IgM antibodies were reached after about a month of the onset of the initial symptoms. Then, the IgG titers plateaued, and the IgM titers decreased, regardless of RT-PCR results. The IgG and IgM levels did not increase after the post-negative positive RT-PCR results in any of the patients. Our results reinforced that the post-negative positive RT-PCR results may be due to the detection of RNA particles rather than reinfection in individuals who have recovered from COVID-19.

Keywords: Reinfection; COVID-19; Severe acute respiratory syndrome coronavirus 2

Coronavirus disease 2019 (COVID-19) is a global pandemic that has spread to 215 countries, resulting in more than 21 million cases and over 760,000 deaths worldwide (data as on August 16, 2020) [1]. In South Korea, strategies to contain COVID-19include extensive testing, epidemiological tracing, and quarantine. However, the recent rise in the number of patients with COVID-19 who have tested positive for severe acute respiratory syndrome coronavirus 2 (SARSCoV-2), via the reverse-transcription polymerase chain reaction (RT-PCR) test, after recovery has raised questions regarding the medical measures and policies. According to the recent report by Korea Centers for Disease Control and Prevention (KCDC) [2], no secondary COVID-19 cases were identified by epidemiological investigations of 790 contacts linked to 285 patients (out of 447 patients, 63.8\%) that tested post-negative positive for SARS-CoV-2 by RT-PCR test (as of May 15, 2020). However, the dynamics of antibody responses and viral load in patients with positive RT-PCR results after recovery from primary infection with SARSCoV-2 have not been reported.

\footnotetext{
*'These authors contributed equally to this work.
} 
We searched the medical records of all COVID-19 patients hospitalized at Seoul National University Bundang Hospital in Seongnam, Korea, and reviewed the records of the patients with positive RT-PCR test results after recovery, including epidemiological data, symptoms, signs, and the results of RT-PCR tests. The humoral immune response against SARS-CoV-2 was determined by indirect enzyme-linked immunosorbent assay based on recombinant nucleocapsid protein for the detection of immunoglobulin $\mathrm{G}$ (IgG) and immunoglobulin $\mathrm{M}$ (IgM) antibodies. Methods used for quantification of the antibodies are described in the supplementary material. This study was approved by the Institutional Review Board of Seoul National University Bundang Hospital (IRB No. B-2006-618-306). The Institutional Review Board waived the requirement for informed consent.

Four patients had positive RT-PCR results for SARSCoV-2 after discharge. Patient 1 was a 73-year-old woman diagnosed with COVID-19 after exposure to her son and daughter-in-law, who had COVID-19. She presented with a 2-day history of fever and a 1-day history of headache, cough, and a sore throat. The other three patients were a family, which consisted of a 33-year-old man (Patient 2), his 31-year-old wife (Patient 3), and their 18-month-old daughter (Patient 4). Three days after exposure to a patient with COVID-19, Patients 2 and 3 developed fever and a productive cough, and were diagnosed with COVID-19. At that time, Patient 4 was asymptomatic; however, she was screened for SARS-CoV-2 infection and tested positive. The day after diagnosis, she became febrile.

Patient 1 was diagnosed with COVID-19 pneumonia and subsequently treated with lopinavir/ritonavir for 10 days. The other three patients did not receive any antiviral drug treatment. All patients were hospitalized and placed in isolation for 13 to 29 days. Clinical symptoms and signs resolved at least 6 days (range, 6 to 20) prior to discharge. All patients had two consecutive negative RTPCR test results at least 24 hours apart before discharge, according to the national guidelines.

Four days after discharge, Patient 1 developed a productive cough and was retested 2 days later, and the result was positive. Patient 4 developed a $37.7^{\circ} \mathrm{C}$ fever and a productive cough after 9 days of discharge and was retested; the RT-PCR test result was positive. Although her parents (Patients 2 and 3 ) had no symptoms, they were retested on the same day as their daughter, and both tested positive.

Patient 1 was tested for other respiratory viruses using a multiplex RT-PCR test performed on a bronchoscopic alveolar lavage specimen, but no other viruses were detected. Patient 2, 3, and 4 were also tested for other respiratory viruses using the multiplex RT-PCR test using nasopharyngeal swab specimens. Patient 4 tested positive for adenovirus and bocavirus but her parents tested negative for all viruses. Epidemiological investigations did not reveal any secondary cases of COVID-19 linked to any of the four patients after their first discharge.

The time course of SARS-CoV-2 levels in respiratory specimens and humoral immune response of each patient in relation to their clinical signs, symptoms, and RT-PCR tests are shown in Fig. 1. Although the RNA-dependent RNA polymerase (RdRP) cycle threshold (Ct)-values increased over time in all the four patients, the values fluctuated. In Patient 2, negative tests were confirmed twice after the first post-negative positive result. He showed a second post-negative positive RTPCR test result 2 weeks after his first post-negative positive test result. In all patients, the IgG levels were the highest 1 month after the onset of the initial symptoms, and they did not increase after the post-negative positive RT-PCR test results were confirmed. Patient 2 did not show an increase in the IgM levels, but in the other three patients, the IgM levels reached their maximum about a month after the onset of the first symptoms and then decreased. The IgG and IgM levels did not rise after the post-negative positive results in any of the patients.

Other studies have shown that in patients who are asymptomatic at the time of the post-negative positive RT-PCR test, the humoral response may be weak and may develop gradually. However, IgM and IgG become detectable in all COVID-19 patients between the 3 rd and $4^{\text {th }}$ week of the onset of their clinical illness [3,4]; thereafter, IgM levels begin to decline and reach lower levels by the 5 th week, while IgG antibodies persist beyond the 7th week [5]. In this study, IgG and IgM antibodies persisted in all patients, but there was no increase in the antibody titer after more than 4 weeks of the onset of the first symptoms. These results suggest that the redetection the virus is more likely to have been due to the presence of RNA particles in respiratory samples, rather than re-infection or reactivation of the live viruses. 

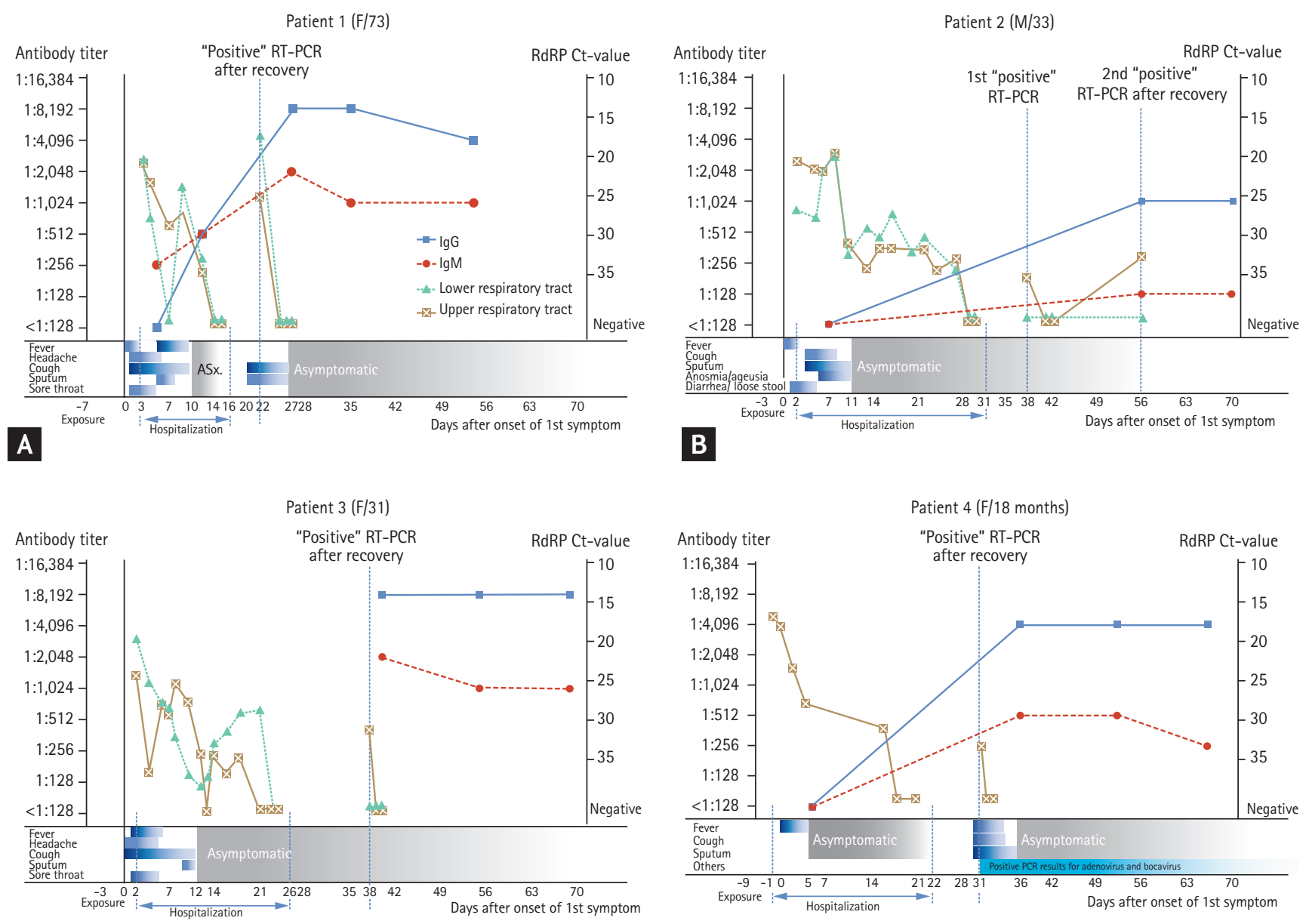

C

D

Figure 1. Viral kinetics and humoral immune response in four patients with repeat-positive reverse transcription polymerase chain reaction (RT-PCR) results for severe acute respiratory syndrome coronavirus 2 (SARS-CoV-2) after recovering from COVID-19. (A) Patient 1: a 73-year-old woman; (B) Patient 2: a 33-year-old man; (C) Patient 3: a 31-year-old woman (the wife of Patient 2); and (D) Patient 4: an 18-month-old girl (the daughter of Patients 2 and 3). The gray lines represent the viral load levels; the solid blue lines represent the immunoglobulin $\mathrm{G}(\mathrm{IgG})$ trajectory; and the dashed blue lines represent the IgM trajectory. Solid grey triangles indicate that the specimen was an upper respiratory sample, and clear squares indicate that the specimen was a lower respiratory sample. RdRP, RNA-dependent RNA polymerase; Ct, cycle threshold.

There are other studies that have reported patients with post-negative positive SARS-CoV-2 RT-PCR results days or weeks after recovery with previous negative results $[6,7]$. However, RT-PCR tests cannot differentiate infectious viruses from non-infectious RNA [8], and RNA detection does not necessarily signify that complete virus or active infection is present [9]. Prolonged "shedding" may reflect a lack of elimination of the nucleic acid from the tissues [8]. The report [2] from the KCDC showed negative test results in 108 cases after performing viral cell culture of respiratory specimens at the time of second diagnosis. In 23 cases, the serum samples of the first and second diagnoses were tested for the presence of neutralizing antibodies, and $96 \%$ of the cases tested positive. Further, in an animal (rhesus macaques) study, SARS-CoV-2 reinfection failed to demonstrate viral replication in all sites examined, suggesting that SARS-CoV-2 induced protective immunity against re-exposure [10].

Due to the retrospective nature of the study, it had the following limitations. The samples were not collected at fixed time-points because they were collected for clinical, and not research purposes. Stored samples were used to detect antibodies, so storage could have affected 
the test results. Further, we did not perform viral cultures or neutralizing antibody tests. However, based on previous studies and the antibody response during the primary infection and post-negative positive RT-PCRresult period in the four patients, our results suggest that the post-negative positive RT-PCR results may be due to the detection of RNA particles rather than re-infection in individuals who have recovered from COVID-19.

\section{Conflict of interest}

No potential conflict of interest relevant to this article was reported.

\section{REFERENCES}

1. World Health Organization. Novel coronavirus (2019$\mathrm{nCoV})$ situation reports [Internet]. Geneva (CH): WHO, c2020 [cited 2020 Aug 16]. Available from: https://www. who.int/emergencies/diseases/novel-coronavirus-2019/ situation-reports/.

2. Korea Centers for Disease Control and Prevention (KCDC). Findings from Investigation and analysis of re-positive cases [Internet]. Osong (KR): KCDC, c2020 [cited 2020 Jun 9]. Available from: https://www.cdc.go.kr/ board/board.es? $\mathrm{mid}=\mathrm{a} 30402000000 \&$ bid=0030\&act=view \&list_no $=367267 \&$ nPage $=1 /$.

3. Sethuraman N, Jeremiah SS, Ryo A. Interpreting diagnostic tests for SARS-CoV-2. JAMA 2020;323:2249-2251.

4. To KK, Tsang OT, Leung WS, et al. Temporal profiles of viral load in posterior oropharyngeal saliva samples and serum antibody responses during infection by SARSCoV-2: an observational cohort study. Lancet Infect Dis 2020;20:565-574.

5. Xiao AT, Gao C, Zhang S. Profile of specific antibodies to SARS-CoV-2: the first report. J Infect 2020;81:147-178.

6. Li J, Zhang L, Liu B, Song D. Case report: viral shedding for 60 days in a woman with COVID-19. Am J Trop Med Hyg 2020;102:1210-1213.

7. Lan L, Xu D, Ye G, et al. Positive RT-PCR test results in patients recovered from COVID-19. JAMA 2020;323:15021503.

8. Atkinson B, Petersen E. SARS-CoV-2 shedding and infectivity. Lancet 2020;395:1339-1340.

9. Alvarez-Moreno CA, Rodriguez-Morales AJ. Testing dilemmas: post negative, positive SARS-CoV-2 RT-PCR: is it a reinfection? Travel Med Infect Dis 2020;35:101743.

10. Chandrashekar A, Liu J, Martinot AJ, et al. SARS-CoV-2 infection protects against rechallenge in rhesus macaques. Science 2020;369:812-817. 